\title{
Classification of Marble Using Image Processing
}

\author{
Fisha Haileslassie ${ }^{1, ~ *}$, Adane Leta ${ }^{2}$, Gizatie Desalegn $^{1}$, Meles Kalayu ${ }^{3}$ \\ ${ }^{1}$ Department of Computer Science, Faculty of Technology, Debre Tabor University, Debre Tabor, Ethiopia \\ ${ }^{2}$ Colleage of Informatics, University of Gondar, Gondar, Ethiopia \\ ${ }^{3}$ Department Information Technology, Raya University, Maichew, Ethiopia
}

Email address:

fishhaile2721@gmail.com (F. Haileslassie)

${ }^{*}$ Corresponding author

\section{To cite this article:}

Fisha Haileslassie, Adane Leta, Gizatie Desalegn, Meles Kalayu. Classification of Marble Using Image Processing. International Journal on Data Science and Technology. Vol. 5, No. 3, 2019, pp. 57-65. doi: 10.11648/j.ijdst.20190503.11

Received: November 13, 2019; Accepted: December 18, 2019; Published: December 24, 2019

\begin{abstract}
Classification of marble image according to usage purpose and quality is an important procedure for export. Discrimination between marble varieties is a difficult task during selection, since it requires trainings and experience. Therefore, the development of automatic prediction model based on image processing is a potential application area to support experts across the world. In this study an attempt has been made to develop marble variety classification model by comparing color, texture and ensemble of color and texture. In view of this, a digital image processing technique based on combined texture and color features have been explored good classification performance to classify varieties of marble image. On the average 60 images were taken from each of the three marble varieties (Grade A, Grade B, Grade C). The total number of images taken was 180 . For the classification model we applied image preprocessing techniques; image acquisition, image conversion, noise removal, image enhancement, edge detection and image binarization. For texture extraction gray level cooccurrence matrix, for color extraction color histogram was applied. For classification five textures and six color features were extracted from each marble image. To build the classification models for prediction of marble varieties, K-Nearest Neighbors $(\mathrm{KNN})$, Artificial Neural Network (ANN) are investigated. Based on experimental results, ANN outperforms KNN. Quantitatively, an average accuracy of $83.3 \%$ and $93.7 \%$ is achieved KNN and ANN respectively for Grade A, Grade B, Grade $\mathrm{C}$ varieties with the combined feature sets of color and texture. This shows an encouraging result to design an applicable marble classification model. Marble fractured and vines of the images affect greatly the performance of the classifier and hence they are the future research direction that needs an investigation of generic noise removal and feature extraction techniques.
\end{abstract}

Keywords: Classification of Marbles, Feature Extraction, Artificial Neural Network Classifier, K-nearest Neighbor, Sobel Edge Detector

\section{Introduction}

Image processing deals with manipulation of digital images through a digital computer for analyzing image data; it is the realization of almost all the services of the human visual system through computers [1]. It is a type of signal dispensation, which outputs an image or characteristics associated with that image. The main objectives of image processing are to create more suitable images for people to observe, identify and, computers can automatically recognize and understand images [1].

Image-processing technology, has rapidly developed since the 1960 s and 1970s [1, 2]. It is basically the technique of manipulating and improving gray-scale images by using mathematical functions. Image-processing system contains three fundamental elements: an image-acquisition element, an image-processing element, and an image-display element. Image acquisition is the process of obtaining a digital image of object in real world using camera or scanner [1]. This image processing element are image enhancement, image analysis, and image-coding $[1,2]$. For human viewing, image enhancement improves the quality and clarity of images. Image analysis involves calculations on a final image to produce numerical results. Image analysis in general is to extract, from the very large amount of data in an image, that small set of measurements containing the information of 
interest [1]. The standard strategy to achieve this is to break the whole task into a sequence of smaller, independent steps. Image analysis starts from image preprocessing such as image acquisition, digitization and image processing methods such as edge detection, noise removal, image type conversion, segmentation, binarization, feature extraction, classification and re-calling of images using different numerical sub-methods $[3,4]$. The objective of each step is to achieve a significant amount of data by discarding irrelevant information $[1,2]$.

Image processing was applied in different problem domain for long time and very recently it has been widely applied in marble mineral mining industries for the purpose of quality grading and classification. The marble production system begins in a quarry with the quarrying of raw natural blocks using different tools and methods [3]. Classification of marble according to usage purpose and quality is an important procedure. But classification and grading of marble products is performed with a human operator by observing physical properties such as structure and color. Physical properties of marble such as hardness, grain size, fracture, streaks, porosity, size, orientation, thickness, distribution of filled joints, gloss and strength are used as classification criteria's by the geologist experts [6-10].

Obviously, this is not the first work in marble image classification. Many international researches published an automatic marble classification model to facilitate the selection, classification and grading of different marble classes on different production areas [6, 11-13]. Most of the researchers used texture or color for classification criteria. Marble minerals content produced from different areas has different texture content; this is due to the nature of the environment such as temperature, weather condition, pressure, elements that found in the marble, impurities and general environment [13].

Classification of Ethiopian marble is performed by the geological expert by observation of color and texture properties of the image. The existing classification approach is subjective decisions, the visual fatigue after a period of time which degrades the performance of the classification, Due to light conditions offer different visual perceptions of a same pattern at different times. The accuracy of the classification procedure is low inconsistent, error prone, time consuming and inefficient because it is manually determined by different level skills experts. There is no machine learning to classify either the marble stone is class A, B or C. Due to this, it has a negative impact on customer satisfaction, since the price of marble types also varies drastically from one another. In this regard, the intention of this research is to design and implement Ethiopia marble image classification by comparing texture, color and the ensemble of color and texture by applying effective pre-processing and feature extraction techniques. For selection of precise quality and types of marble used Artificial Neural Network (ANN) and K-Nearest Neighbor (KNN) classifiers to achieve better classification performance.

\section{Methodology of the Study}

\section{Dataset Preparation}

Dataset preparation is the main procedure for collecting datasets of all the marble class varieties. For this work varieties of marble have been collected from Saba marble factory. In the existing practice experts used fractured, vine, color and texture as classification criteria. From these datasets, $80 \%$ have been used for training and $20 \%$ for test. The images used for this classification system are prepared using a HP camera with 16 megapixels. 180 images are prepared with the same resolution of 600 DPI for image processing. DPI keeps all image with same size of pixel. For this research work, we have been interviewed the domain expert's using purposeful sampling technique to collect the required information how they used to classify about marble varieties. Moreover, personal observation, and record reviews also served as data source and collection methods.

Image preprocessing

For this study we follows the standard image preprocessing approaches to process image acquisition, color conversion, noise removal, image enhancement, binarization, for feature extraction GLCM and color histogram algorithms and for pattern classification ANN and KNN algorithm used to provide decision. So, in this study we determined for preprocessing techniques are median filtering to filter noise such as Gaussian, salt and pepper, speckle and Poisons (Shot noise) in the image because these noises are commonly happened during image acquisition. True color conversion in to gray scale is used to minimize computational difficulty or to reduce the number of representing values. The most commonly used color conversion techniques are lightness, average and luminosity. Image enhancement techniques help in improving the visibility of any portion or feature of the image suppressing the information in other portions or features. Techniques commonly used for image enhancement is histogram equalization and contrast enhancement. Image Edge detection significantly reduces the amount of data and filters out useless information. the commonly used edge detecting methods used in image processing are Canny edge and Sobel edge detector operators. For this research Sobel edge detection is effective in identifying true and false edges. Image binarization is a process of separation of pixel values of an input image into two-pixel values; black as foreground and white as background. Otsu's thresholding method is the most commonly used method for image binarization using a global threshold. After preprocessing the image, it has been binarized to identify and extract the features for representation so as to identify marble class for feature extraction to develop a predictive model for classification.

Feature Extraction

Feature extraction is a method of capturing visual content of images for classification and retrieval. image analysis is used for extracting meaningful information from images that are used for classification of images to different categories. For image analysis of marble three classification parameters are identified. They were texture features, color features and 
the combination of color and texture. The commonly used texture feature extraction techniques are The Gray Level Cooccurrence Matrix (GLCM) and Sum and Difference Histogram (SDH) [1]. Color histogram is used to determine the distribution of colors within a whole or within an interest region of image. For this study gray level co-occurrence matrix technique is used because it is easy to implement and reduces the computation time [14]. for this study five (contrast, energy, correlation, homogeneity and entropy) texture and six (red, green, blue, hue, intensity and saturation) color features were extracted from the image.

Image classification model

To implement the proposed model for the classification of marble image based on color, texture and the combination of color and texture, neural network classification and K-nearest neighbor approaches were compared. For the neural network classification, feed forward multilayer neural network model was used.

Tools Used

The sample marble image taken be processed using a personal computer with a specification of Intel (R) core (TM) i5_3110M CPU of $2.5 \mathrm{GHz}$ processor speed and $4.00 \mathrm{~GB}$ (RAM), 800 GB hard disk capacity with Microsoft window 7 professional 64 Operating system. MATLAB software version R2015a computing environment is used for implementation. MATLAB is a multi-paradigm numerical computing environment [15]. It allows the use of a set of predefined functions called Toolboxes and the user defined functions and programs. MATLAB Neural Network Toolbox provides tools for designing, implementing, visualizing, and simulating neural networks for various applications [15]. MATLAB facilitates the user to develop applications using graphical user interface (GUI) or command line interface (CLI).

\section{Model Evaluation}

The collected dataset has been divided into training and testing, the training data is used to develop models of marble classification and analysis, while the test data is used to validate the performance of models from training data. The performance of the model, built for marble classification, is measured based on the number of correctly and incorrectly identified marble varieties in terms of accuracy and error rate. Accuracy is a measure of how closely the experimental results agree with a true or accepted value. It can be calculated as the ratio of correctly predicted values to total number of prediction while error rate is the ratio of wrongly predicted value out of the total number of predictions [16].

\section{Experimentation}

In this study, an attempt is made to develop a model for the classification of marble using image processing techniques. Detailed record preparation, implementation, experimental results and challenges are presented in this chapter.

Dataset Preparation

Since there is no ready-made dataset for this type of research, we have prepared dataset for training and performance evaluation. To do this, marble image have been collected from Saba marble Factory in Ethiopia. The collected marble images are certified by the domain experts by physical appearance observing texture and color. To get clear image, it has been performed with vertical and horizontal resolution of 600 dot per inch that means to use the same pixel value of the image and stored in JPG format. The details quantities and varieties of sample marble images used in the experimentation is summarized in Table 1 below.

Table 1. Summary of marble image collected for the current study.

\begin{tabular}{lllll}
\hline Marble image & \multicolumn{4}{l}{ Description } \\
\hline Number of images each Varity & Class A & Class B & class C & Total \\
& 60 & 60 & 60 & 180 \\
Image format & JPG & & & \\
Image resolution & $600 x 600$ DPI & & \\
Digitization acquisition & HP Digital camera with & 16 megapixels \\
\hline
\end{tabular}

Grayscale Conversion

for this study it converts the color image of the captured marble image into grayscale (image which only has a bit value). For the implementation, use the default, MATLAB Image Processing Toolbox, image type conversion function called rgb2gray (I). The rgb2gray (I) function converts the RGB I image to grayscale by eliminating hue and saturation information while maintaining luminance using a weighted sum of the R, G, and B components. The sample image which is in RGB format and its equivalent gray level image which are prepared for subsequent processes are shown below in figure 1 (a) and (b) respectively.

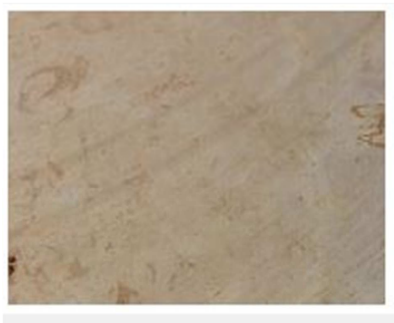

(a)

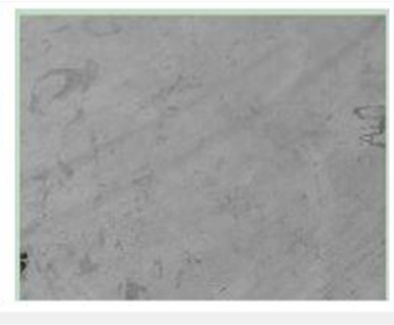

(b)
Figure 1. Sample Class A marble image; RGB image converted into Grayscale image.

\section{Noise Removal}

To clean noise from the images, we have tried to use other noise filtering algorithms such as average filtering but from the experimental result using median filtering algorithm enhances the image clearly. So for this study we consider the median filtering techniques since it offers advantages: no reduction of contrast across the stages, it does not push boundaries, it is less sensitive than the average to extremes or outliers, it preserves edges while removing noise, and for certain types of random noise they provide excellent noise reduction capabilities with significantly less Blurring about the others $[17,18]$. It is also good enough to remove impulsive (salt-and-pepper) noise [19, 20].

The median filter is implemented using a MATLAB function. Medfilt2 (I, [m n]) performs a median filtering of a 
gray image I, where each output pixel contains the median value in the $m$ by $n$ neighborhood. Medfilt2 () buffers the image with $0 \mathrm{~s}$ at the edges, so that the median values for the points within $[\mathrm{m} \mathrm{n}] / 2$ of the edges appear distorted. The sample marble image, after the implementation of median filtering, in gray level format and its equivalent filtered image which are prepared for subsequent processes are shown below in Figure 2 (a) and (b) respectively.

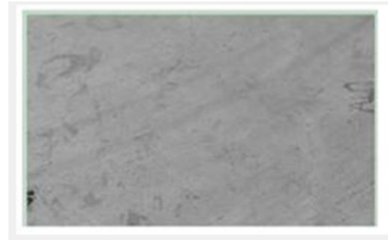

(a)

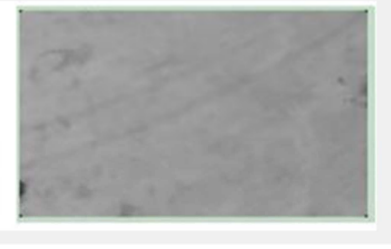

(b)
Figure 2. Sample Class A marble image; (a) gray level before median filter applied, (b) gray level after median filter.

\section{Image Contrast Enhancement}

Image enhancement operations typically return a modified version of the original image and are frequently used as a preprocessing step to improve the results of image analysis techniques. This is done using MATLAB function $\mathrm{I}=$ imhist () to display the histogram of an input image and $\mathrm{j}=$ histeq () to perform histogram equalized or enhanced marble image. Histogram of input image before and after contrast enhancement is shown in the figure 3 below.

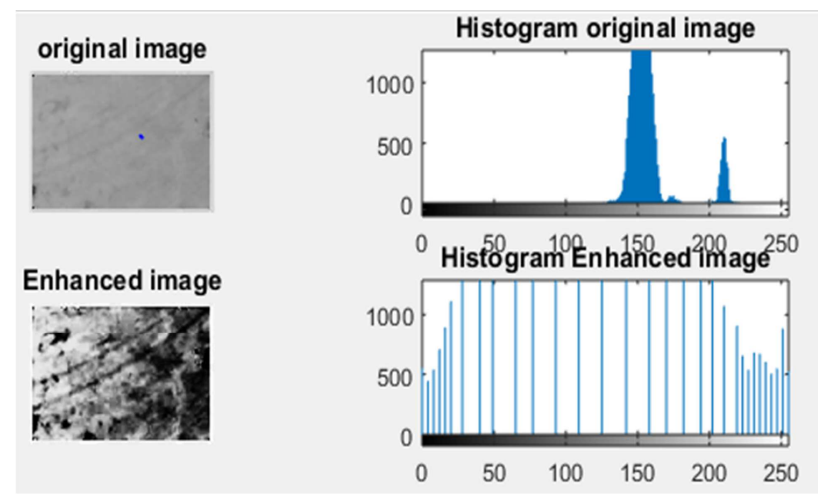

Figure 3. Histograms of an input and its contrast enhanced marble images.

\section{Binarization}

After applying a preprocessing algorithm of the marble image, converted the filtered grayscale marble image to a binary ( $0 \mathrm{~s}$ and $1 \mathrm{~s})$ format binarization.

Therefore, in this study, for binarization, it implements Otsu thresholding techniques. for an input filtered grayscale image having a threshold $\mathrm{T}$, the pixels 1 (white) when the gray level is greater than the threshold $\mathrm{T}$ will otherwise become 0 (black).

The Otsu binarization technique is implemented using the built-in MATLAB graythresh () function. This function calculates a threshold that can be used to convert binary image with the function im $2 \mathrm{bw}$ (). A threshold is normalized and breast value is between zero and one [1]. The function $\mathrm{bw}=\mathrm{im} 2 \mathrm{bw}(\mathrm{I}$, level) converts the filtered grayscale image I into a binary image.

The sample binarized marble image, after the implementation of Otsu thresholding technique, on filtered gray level format is shown in Figure 4 below (a) and (b) respectively.

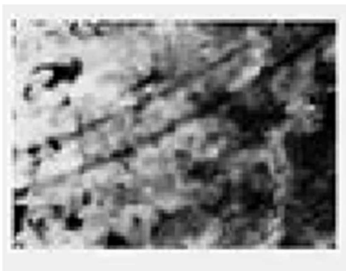

(a)

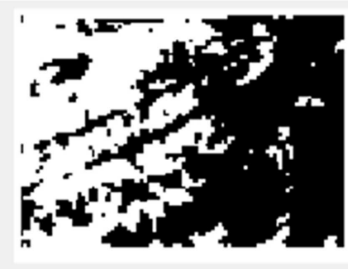

(b)
Figure 4. Binarizations of input marble images.

Edge detection

In this work used Sobel edge detection because it provides sharper and clearer edges compared to other operators and is easy to use. To implement this method, a MATLAB function edge (BW, 'Sobel') is used. The Sobel method uses the derived approximation to find edges. Therefore, there are edges at those points where the gradient of the image is considered maximum. The binarized marble image of class A and its equivalent edges using Sobel edge detection are shown figure 5 below (a) and (b), respectively.

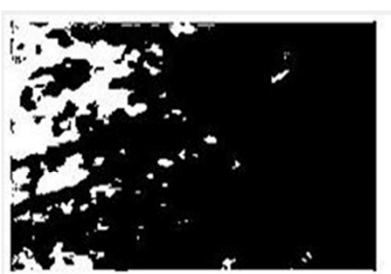

(a)

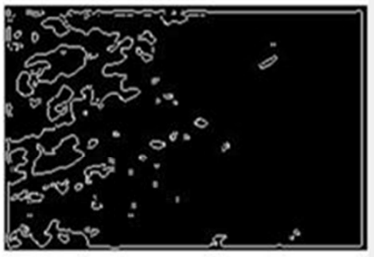

(b)
Figure 5. Edge detection using Sobel operator.

\section{Feature extraction}

The features of images and the extraction method used during experimentation are decided by the output of the image-preprocessing steps. In the traditional system, human vision attempts to identify marble class using structural forms such as shape, size, their visual color differences and intensity variation of their surface. Hence, the current classification model is proposed based on texture and color analysis, which considers an assessment of human visual inspection and physical characteristics of each marble image as starting point. Based on this, we extract 11 features from each marble image. These are contrast, energy, entropy, homogeneity, correlation, red, green, blue, hue, saturation and intensity. To extract features, two feature extraction techniques which are implemented in MATLAB as a function; graycoprops () and $\operatorname{rgb}(:,:$, c) for textural and color feature respectively.

The function graycoprops (GLCM, properties), measures a set of textural properties from the Gray-Level Co-occurrence Matrix GLCM like Contrast, Correlation, Energy, Entropy, 
and Homogeneity. The function rgb $(:,:$, c) computes such as Red, Green and Blue components from the RGB images and the value of Hue, Saturation and Intensity. Then a total of 11 features, from 180 marble images, are extracted.

A General Approach for Building Classification Model

A general model for classification of marble image is depicted in Figure 6. This model can be explained in four phases which are data set partitioning, model building, model testing, and finally we measure classification model performance.

Building Model

As described in Figure 6 below, our classification model is constructed by feeding the training data set which was partitioned in data set partitioning phase. In order to build the model, it used Artificial Neural Network (ANN) and Knearest neighbor (KNN) as classification algorithms.

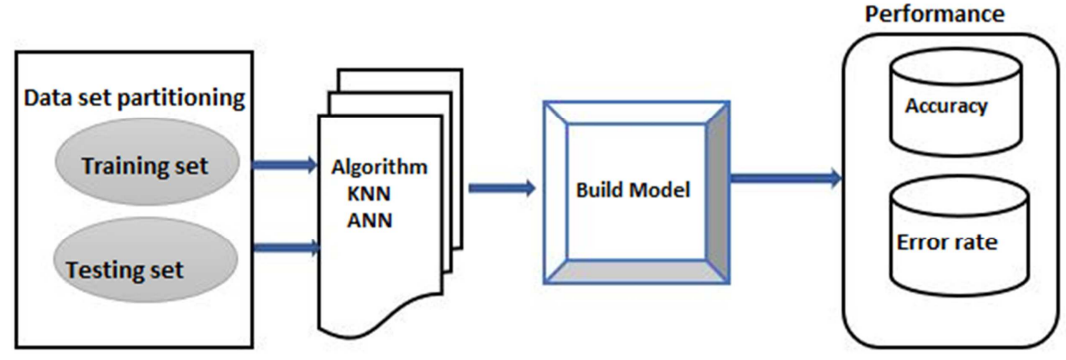

Figure 6. General Approaches for marble classification model, adopted [5].

Experimental result

Once the predictive model is developed an experiment is conducted to evaluate its performance in the classification of marble image varieties. To this end, experiments are conducted for each classifier using color, texture and the combination of color and texture features. In each experiment the classification algorithms; MLP, KNN are used so as to select the best performer for classification of marble image verities. In this study, first it extracted 5 textural and 6 color features from the binarized marble image. These extracted features are used for both K-nearest neighbor and Artificial neural network to classify where it the class belongs.

Artificial Neural Network Classifier

The first classification algorithm for constructing marble image variety classification model is artificial neural network. The parameters used for the classifier with their value are depicted in table 2 .
Table 2. The performance of ANN model for the selected feature sets.

\begin{tabular}{ll}
\hline Parameters & values \\
\hline Number of hidden layers & 10 \\
Network type & Feed-forward with backpropagation \\
Number of neurons in hidden layer & 6 \\
Activation function & Sigmoid \\
Algorithm & Multilayer perceptron \\
Initial weight & 1 \\
Initial bias & 1 \\
\hline
\end{tabular}

Parameters used for ANN classifiers

The features extracted, from marble images, using the feature extraction techniques discussed so far are then, feed to the artificial neural network classifier to measure the accuracy for each technique. The accuracy of each technique is then measured, by dividing correctly identified marble image varieties to the total number of marble image varieties, depicted in table 3 .

Table 3. The performance of ANN model for the selected feature sets.

\begin{tabular}{llll}
\hline Features extracted & Correctly identified & Incorrectly identified & Accuracy (\%) \\
\hline Texture & 42 & 18 & 68 \\
Color & 46 & 13 & 76 \\
Color + Texture & 56 & 4 & 93.7 \\
\hline
\end{tabular}

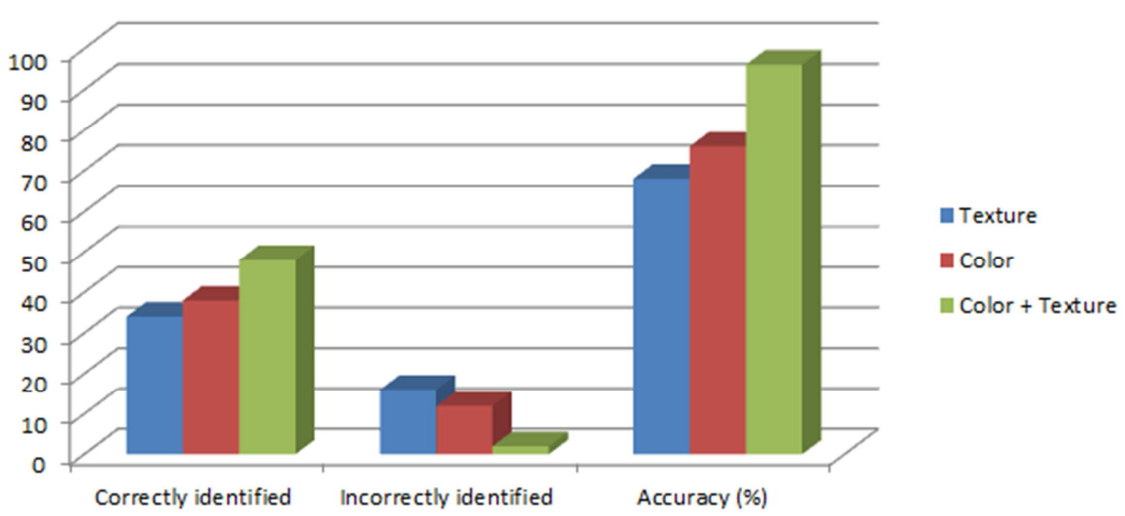

Figure 7. The performance of each feature set using ANN model. 
As can be seen from the above table combined feature set of texture and color registered better results than the other feature set with an accuracy of 93.7\%. From this experimental result we have observed, using the combined features of color and texture neural network model showed relatively better discrimination result than using individual features. Classification using color feature is better than texture feature because color of the images can be isolated certainly but texture properties are challenging to identify they have comparable properties. The experimental results achieved using these feature sets are presented using a confusion matrix as follows in Table 3.

A confusion matrix for the combination of texture and color features using ANN: In this experiment, our neural network model used 11 (combination of textures and color) features. Hence, the neural network model has 11 inputs, 10 hidden and 3 output units (11:10:3) where input units are equal to the number of features and the outputs are the number of class. The results achieved during experimentation are summarized and shown in table 4.

Table 4. The performance of ANN model for the selected feature sets.

\begin{tabular}{llllll}
\hline Predicted Class & Actual Class & Grade A & Grade B & Grade C & Total (\%) \\
\hline Grade A & & 58 & 2 & 0 & 96.6 \\
Grade B & 0 & 53 & 7 & 88.3 & 3.3 \\
Grade C & & 3 & 57 & 95 & 11.7 \\
total\% & Correctly & 96.7 & 88.3 & 95 & 93.7 \\
\hline
\end{tabular}

Based on this experimental result $93.7 \%$ were correctly identified and $6.3 \%$ of the sample data set images were incorrectly identified. An investigation of the results indicated that 2 image of class A were incorrectly identified as Class B. 7 image of class B misidentified as class C. 3 image of Class $\mathrm{C}$ were incorrectly identified as Class B. From this experimental result we have observed that class $\mathrm{A}$ varieties are better discriminated the main reason for confusion is due to the existence of texture structure and color distribution similarities among those misidentified varieties. From this experimental result there is no correlation between Class A and Class C.

According to experimental result summarized in table 4, the correlation between each of the four varieties of marble is depicted using a bar chart in Figure 8.

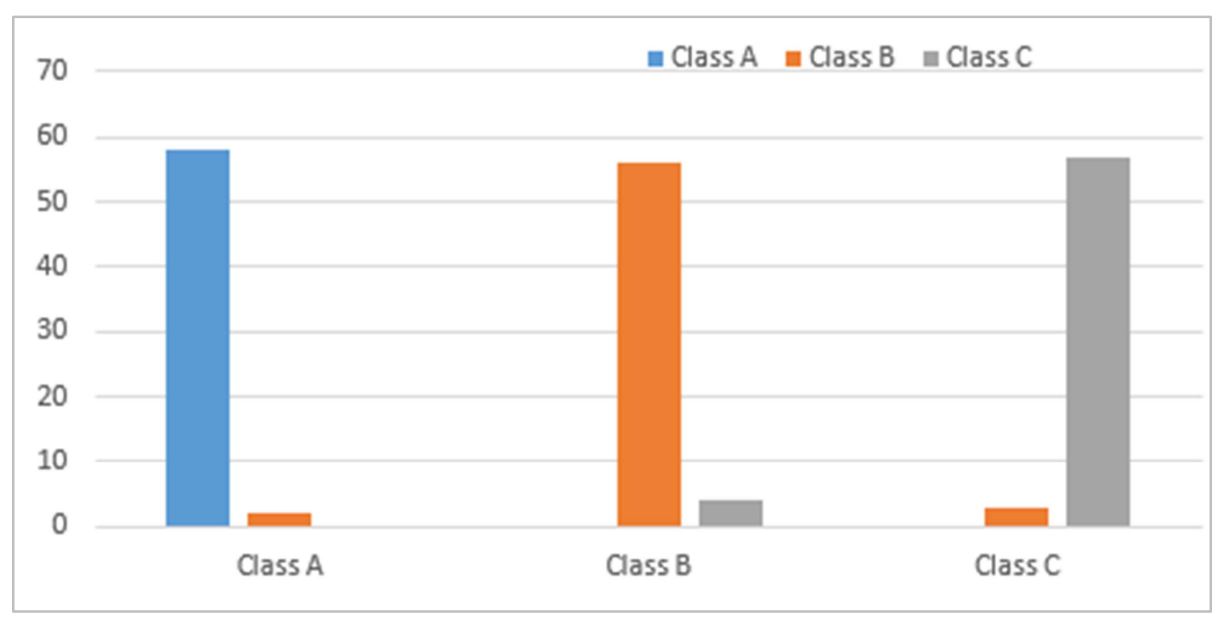

Figure 8. Correlation of the class using ANN model.

The correlation chart in Figure 8 indicates that Class A varieties are more correlated with Class $\mathrm{B}$. As we observe the Class $\mathrm{C}$ varieties more correlated with class $\mathrm{B}$ and class $\mathrm{C}$ are stand alone, it shows that as no correlation between class $\mathrm{A}$ and class $\mathrm{C}$.

\section{K-Nearest Neighbor Classifier}

In the case of K-nearest neighbor classification, the training data set which is used by neural network so far, both the input and their target variables are used to build the model. And then the class of the test data, which contain only the input variables, is determine by comparing the distance of the unknown tuple to $\mathrm{K}$ nearest neighbors of that reference model. We consider Euclidean distance as the distance metric parameters while 3 is the value of $\mathrm{k}$. Based on this, the accuracy of each feature set is depicted in table 5 .

Table 5. The performance of KNN model for the selected feature sets.

\begin{tabular}{llll}
\hline $\begin{array}{l}\text { Features } \\
\text { extracted }\end{array}$ & $\begin{array}{l}\text { Correctly } \\
\text { identified }\end{array}$ & $\begin{array}{l}\text { Incorrectly } \\
\text { identified }\end{array}$ & $\begin{array}{l}\text { Accuracy } \\
(\%)\end{array}$ \\
\hline Texture & 44 & 16 & 73.2 \\
Color & 45 & 15 & 75 \\
Texture + Color & 51 & 9 & 83.3 \\
\hline
\end{tabular}




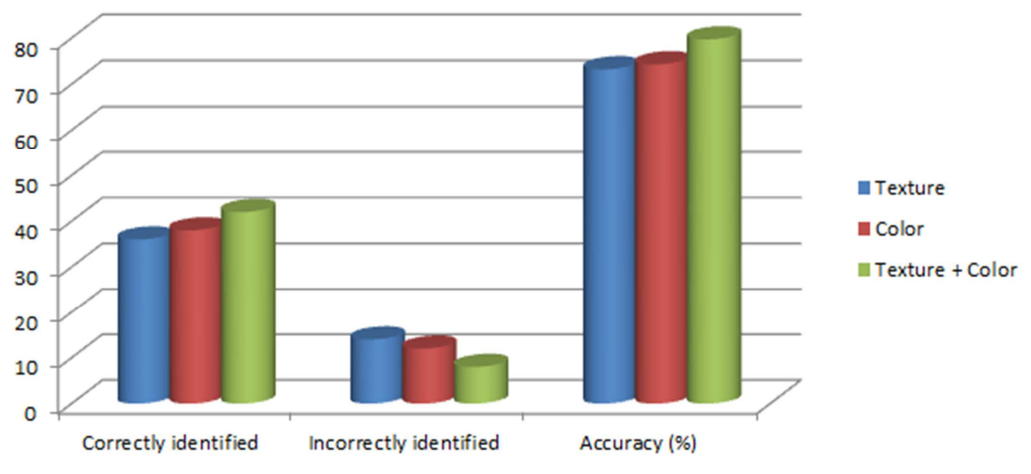

Figure 9. Tthe performance of each feature set using KNN model.

Based on the experimental results summarized in table 5, the combined feature sets of texture and color features registered better results than the other feature set with an accuracy of $83.3 \%$. The comparative classification model of color and texture, using color feature archives better result than using texture classification. The reason to vary the classification accuracy is having the same challenges as we discussed in ANN. As we have been observed from this experimental result using the combined features achieved good result, the reason for better classification is combination of color and texture feature it describes the properties of the marble image.

A confusion matrix for the combination of texture and color features using KNN: During this experiment we have been used a combination of all the two features employed in our experimentation. The results registered during experimentation are summarized and shown in table 6 .

Table 6. Summary results of marble image using KNN with a combination of color and texture features.

\begin{tabular}{|c|c|c|c|c|c|c|}
\hline \multirow{2}{*}{ Predicted Class } & \multirow{2}{*}{ Actual Class } & \multirow{2}{*}{ Grade A } & \multirow{2}{*}{ Grade B } & \multirow{2}{*}{ Grade C } & \multicolumn{2}{|l|}{ Total (\%) } \\
\hline & & & & & Correctly & Incorectly \\
\hline Grade A & & 52 & 8 & 0 & 86.7 & 13.3 \\
\hline Grade B & & 3 & 50 & 7 & 83.3 & 16.7 \\
\hline Grade C & & 0 & 12 & 48 & 80 & 20 \\
\hline \multirow{2}{*}{ total\% } & Correctly & 86.7 & 71.4 & 85.7 & \multirow{2}{*}{83.3} & \multirow{2}{*}{16.7} \\
\hline & Incorrectly & 13.3 & 29.6 & 14.3 & & \\
\hline
\end{tabular}

Based on this experimental result, out of 60 images, $83.3 \%$ were correctly identified and $16.7 \%$ of the sample data set images were incorrectly identified. An investigation of the results indicated that 8 images in each of Class $\mathrm{A}$ were incorrectly identified as Class B. 3 image from Class B and 7 images from Class B were incorrectly classified as Class A and C. 12 image class C were incorrectly classified as class
B. From this experimental result we have observed that none of the varieties are perfectly discriminated while Class A varieties are the best discriminated and also Class $\mathrm{B}$ is more correlated with Class $\mathrm{C}$. there is no correlation between Class $\mathrm{A}$ and $\mathrm{C}$. The correlation between each of the three varieties of marble image is depicted in Figure 10.

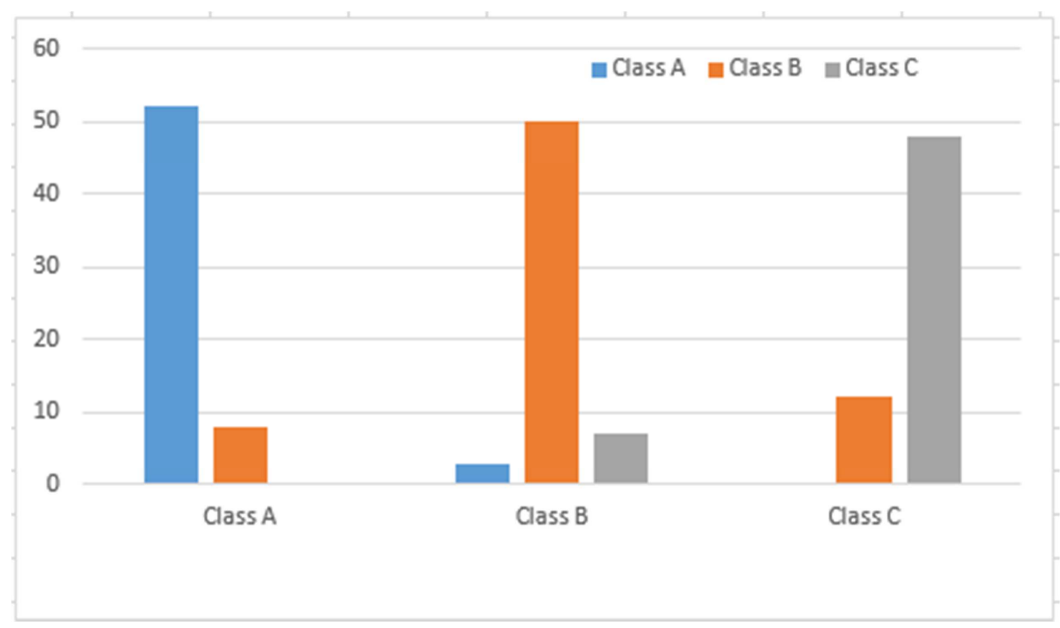

Figure 10. Correlation of the class using KNN model. 
The Overall Performance of Identification Model

As we have presented the detail in the previous sections, the experiments were conducted under three situations by using feature sets of texture, color and combining color with texture. Accordingly, experimental results show that texture feature has the largest discriminating power with neural network while color is better with nearest neighbor.

The best accuracy of neural network model is $93.7 \%$ using combined features of Texture and color because it describes the properties of the image, while K-nearest neighbor achieved $84.3 \%$, Hence, using combined feature of marble image classification shows a better result than using them separately. The classification performance of artificial neural network was better than that of k-nearest neighbor, because ANN can tolerant noise inputs and of representing the Boolean function independently of the others and it depends on the network structure, the pulse rate, the learning rate and the converging criteria, In KNN the cost of the learning process is zero; no assumptions about the characteristics of the concepts to learn have to be done. The classification performance of artificial neural network was better than that of k-nearest neighbor as indicated in figure $11 \mathrm{a}, \mathrm{b}$.

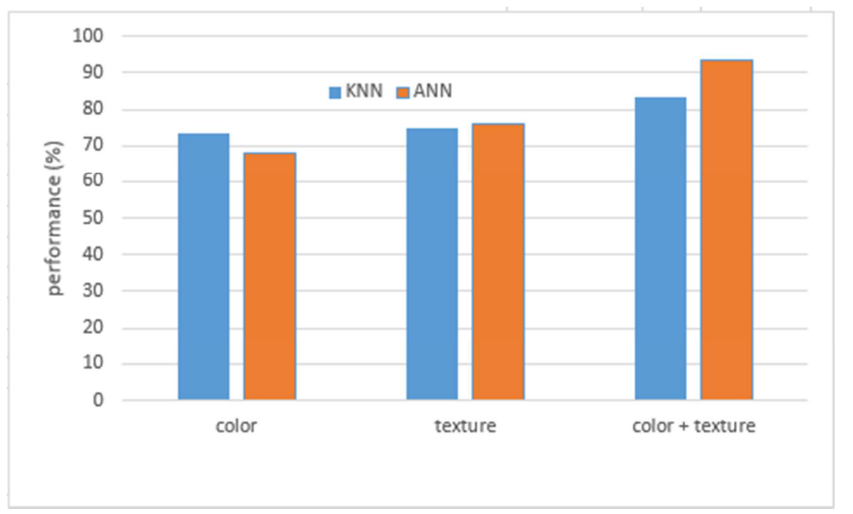

(a) Model performance in each class of marble image

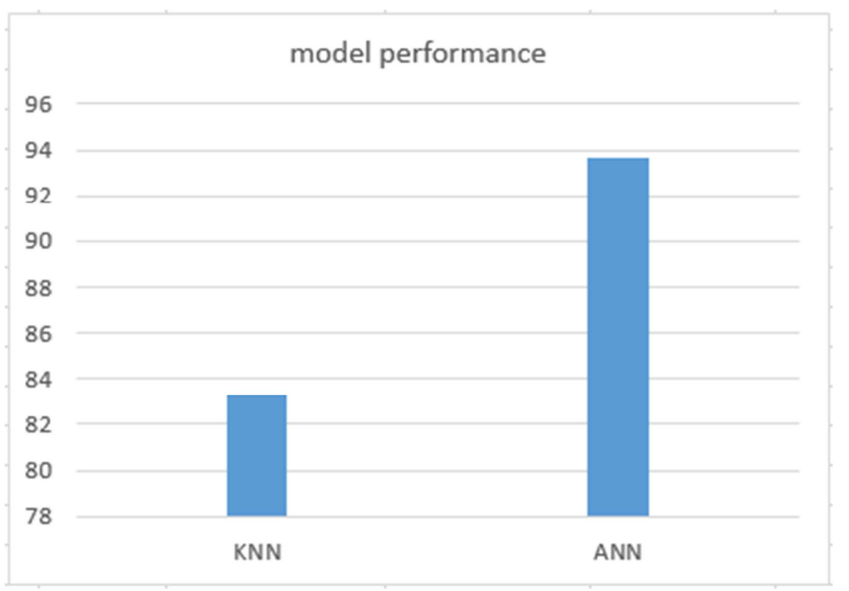

(b) overall performance of models

Figure 11. Model performance comparisons.

\section{Discussion of Results}

As to the knowledge of the researchers, we didn't find previous research works on marble classification country wise in Ethiopia. So, we focus on the dataset type used, segmentation and feature extraction in order to discuss on the results of the system. In this study, we have used Otsu thresholding for segmentation and Grey Level Co-occurrence Matrix and color histogram feature extraction techniques.

In this study, during experimentation color, texture and the combined of color and texture features where used for the classification model by applying ANN and KNN model. During the experimentation we have extracted from the marble image color, texture and finally by combining color and texture to evaluating the performance of the model for both ANN and KNN algorithms. During both KNN and ANN algorithm color improve good performance than texture because colors has good dissimilarity when we came to texture it has very comparable texture similarities. The combined of color and texture improves good performance than color and texture because as we observed from the experiment the combined feature of color and texture well describes the marble image properties.

The comparative analysis achieved by the classification model for the predefined class of marble is, both algorithm ANN and KNN performs good result on the combination of color and texture. When we see the correlation between the pre-defined class, for both classification algorithm from the tested image Grade A has a correlation with Grade B but there is no correlation between with grade $\mathrm{C}$ that means there is no color and texture similarity between Grade A and Grade C. When we came to Grade A and Grade C both grades have a correlation with Grade B the same reason there is similarity in both color and texture. The Grade $\mathrm{C}$ marble have only a correlation with the Grade B only and there is no similarity between Grade A.

As we observed from the experiment, the combination features both color and texture improved the accuracy of the classifier because it describes the properties of the image. Preprocessing error occurred due to the noise removal, binarization and edge detection algorithms. Classification error is occurred due to similarity of color, textural and noise that occurred in the image. For better result this research requires to apply quality of image, advanced noise removal algorithm that improves in detecting blurring and shadow in marble images before applying binarization, feature extraction and classification techniques. so, from this research we understand marble classification using machine learning algorithm is good solution for the experts to identify the grade of the marble with standard procedure.

\section{Conclusion}

Marble is most commonly used for flooring and construction. Marble have different biological and physical characteristics which play the major role in the production of 
marble. Quality of marble with those characteristics used in marble production are selected by the experts with observing physical properties; while this classification expert is bias on the quality classification. The development of marble classification model can support for the selection and classification process of marble. In this study an attempt has been made to construct an optimal model for the classification of Ethiopian marble image verities.

To this end, the study follows experimental research, which involves data set preparation for training and evaluating marble image classification model. Image of Ethiopian marble verities (Grade A, Grade B and Grade C) are captured from Saba marble factory. For this research we have been used image acquisition, image type conversion, noise removal, image enhancement, image binarization at the preprocessing stage. For the color and texture feature extraction we used color histogram and the second order statistical method gray level co-occurrence matrix respectively. After we extracted color and texture features of each variety the identification model is built using ANN, KNN. The test results show that the combined color and texture features are effective in constructing an optimal model for marble verities classification using ANN and KNN with an overall performance accuracy is $93.7 \%$ and $83.3 \%$ respectively. The main challenges for this study are using low quality of camera, noise occur during image collection methods and preparing dataset because there is no readymade dataset. As strength in this study we try to identify different techniques in image analysis for pattern classification and how to design and develop a model for image classification.

\section{References}

[1] T. Acharya and A. K. Ray, Image Processing: Principles and Applications, vol. 15, no. 3. New Jersey, India: A JOHN WILEY \& SONS, MC., PUBLICATION, 2005.

[2] B. K. Behera, Image-Processing in Textiles, vol. 35, no. 2-4. 2004.

[3] Ö. Akkoyun, "An evaluation of image processing methods applied to marble quality classification," 2nd Int. Conf. Comput. Technol. Dev., pp. 158-162, 2010.

[4] B. Jähne, Practical handbook on image processing for scientific and technical applications. 2004.
[5] B. H. BELAY, "Application of Image Processing Techniques for Malt-Barley Seed Identification," Bahir Dar, Ethiop., 2015.

[6] E. Ardal1, "Classification of Marble Textures using Neural Networks and Image Processing Methods," p. 41, 2008.

[7] A. P. D. O. AKKOYUN, "An evaluation of image processing methods applied to marble quality classification," no. January, 2014.

[8] T. Seemann, "Digital Image Processing using Local Segmentation," Philosophy, no. April, pp. 1-300, 2002.

[9] P. J. R. C. Sousa J. M. C., "Comparison of intelligent classification techniques applied to marble classification," Lect. Notes Comput. Sci. (including Subser. Lect. Notes Artif. Intell. Lect. Notes Bioinformatics), vol. 3212, pp. 802-809, 2004.

[10] J. Miguel and P. Batista, "Marble Polished Stones Automatic Classification," pp. 1-8, 2015.

[11] E. Symposium, A. Neural, and N. Bruges, "Marble Slabs Quality Classification System using Texture Recognition and Neural Networks Methodology," no. April, pp. 75-80, 1999.

[12] M. kora. B. Deviren Murat, "A Feature Extraction Method for Marble Tile Classification *," vol. 7, pp. 0-3.

[13] I. B. Gundogdu, "Color identification of some Turkish marbles," vol. 22, pp. 1342-1349, 2008.

[14] S. Gupta and S. G. Mazumdar, "Sobel Edge Detection Algorithm," Internatoinal J. Comput. Sciennce Manag. Res., vol. 2, no. 2, pp. 1578-1583, 2013.

[15] E. J. Leavline, “Artificial Neural Network Design Flow for Classification Problem Using MATLAB," no. December, 2015.

[16] P. Kamavisdar, S. Saluja, and S. Agrawal, "A Survey on Image Classification Approaches and Techniques," vol. 2, no. 1, pp. 1005-1009, 2013.

[17] M. S. Nixon and A. S. Aguado, "Feature Extraction and Image Processing," Acad. Press, p. 88, 2008.

[18] E. I. J. R., Parker, Kostas Terzidis, Algorithms for Image Processing and Computer Processing on Vision Second Edition ed. India, Wiley Publishing, 2011.

[19] M. K. and J. Fridrich, "On Detection of Median Filtering in Digital Images," SPIE Conf. Media Forensics Secur., 2010.

[20] M. Habtamu, "Image Analysis for Ethiopian Coffee Classification," M. Sc. Thesis, Addis Ababa Univ., no. January, 2008. 